\title{
Improved estimates of the physical properties of the O-star binary V1007 Sco = HD 152248 and notes on several other binaries in the NGC 6231 cluster ${ }^{\star}, \star \star$
}

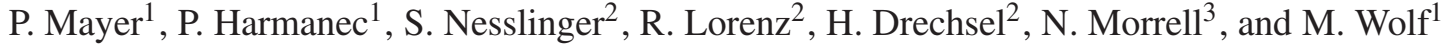 \\ 1 Astronomical Institute of the Charles University, Faculty of Mathematics and Physics, V Holešovičkách 2, 18000 Praha 8, \\ Czech Republic \\ e-mail: mayer@cesnet.cz \\ 2 Dr. Remeis Observatory, University Erlangen-Nürnberg, Sternwartstrasse 7, 96049 Bamberg, Germany \\ ${ }^{3}$ Las Campanas Observatory, Carnegie Observatories, Casilla 601, La Serena, Chile
}

Received 24 August 2007 / Accepted 14 January 2008

\section{ABSTRACT}

\begin{abstract}
Context. In spite of the importance of massive O-type stars for astrophysics, their accurate masses and other fundamental properties are still a matter of debate. Determining them reliably is hampered by various factors (stellar winds and other forms of circumstellar matter), and the agreement of derived properties with the model predictions is far from satisfactory. Careful studies of O-type binaries, especially of those in stellar clusters, are therefore desirable.

Aims. Having obtained new series of electronic spectra and UBV photometry of V1007 Sco, we analysed these data in an effort to check whether the observed properties of V1007 Sco indeed disagree with the prediction of stellar evolutionary models. We briefly analysed data for a few other binaries in NGC 6231, too.

Methods. Spectral reductions were carried out with the MIDAS program, photometry reduced using the HEC22 program, the orbital elements were derived with the FOTEL program and the final solutions obtained with the program PHOEBE.

Results. Our analysis led to an accurate determination of the apsidal advance, $\dot{\omega}=(0.00884 \pm 0.00012)$ deg d $\mathrm{d}^{-1}$, based on a simultaneous solution of all usable radial-velocity and photometric data. This implies an apsidal period of 111.5 years. It is also demonstrated that the orbital inclination must be close to $67^{\circ}$. We arrived at the following preliminary values for masses and radii: $M_{1}=(29.5 \pm 0.4) M_{\odot}, M_{2}=(30.1 \pm 0.4) M_{\odot}, R_{1}=(15.8 \pm 0.7) R_{\odot}$, and $R_{2}=(15.3 \pm 0.5) R_{\odot}$. These values clearly indicate a log $g$ of about 3.5 [CGS], implying that the stars are giants and not supergiants, as the standard spectral classification criteria indicate.
\end{abstract}

Key words. binaries: eclipsing - stars: early-type - stars: fundamental parameters - stars: emission-line, Be stars: individual: V1007 Sco

\section{Introduction}

V1007 Sco (HD 152248, SAO 227382, HIP 82691; $\alpha_{2000}$ : $\left.16^{\mathrm{h}} 54^{\mathrm{m}} 10^{\mathrm{s}}, \delta_{2000}:-41^{\circ} 49^{\prime} 30^{\prime \prime}\right)$ is a member of the open cluster NGC 6231. The star was classified as O7Ib:(n)(f)p by Walborn (1972) and the supergiant classification was confirmed also by Penny et al. (1999) on the basis of optical and far-UV spectra. In our preliminary study we argued, however, that both stars are giants, not supergiants - cf. Mayer et al. (2001). Also Sana et al. (2001) classified the binary as O7.5III(f)+O7III(f).

The spectroscopic duplicity of V1007 Sco was discovered by Struve (1944) on spectra from McDonald Observatory. He was able to measure radial velocities (RVs hereafter) of both components. However, since he could only observe the star near meridian from his location, he arrived at an incorrect period of 3.10. Hill et al. (1974) obtained new RVs and concluded that the true period was $5.97 \pm 0$ d 06 . They derived new circular orbital elements, combining their and Struve's RVs and found a large difference in the $\gamma$ velocities of the two binary components.

\footnotetext{
^ Based on spectral and photometric observations from ESO La Silla and Cerro Tololo observatories.

$\star \star$ Tables 4 and 6 are available only in electronic form at the CDS via anonymous ftp to cdsarc.u-strasbg.fr (130.79.128.5) or via http://cdsweb.u-strasbg.fr/cgi-bin/qcat?J/A+A/481/183
}

Levato \& Morrell (1983) obtained two additonal RVs and by combining them with the velocities of Struve (1944) and Hill et al. (1974) they derived a period of 5d89144 and an eccentric orbit. Using their period, Luna (1988) analysed his polarimetric observations of V1007 Sco and concluded that the orbital inclination had to be between $60^{\circ}$ and $76^{\circ}$.

The photometric variability of V1007 Sco was discovered by Brownlee \& Cox (1953) who observed a light decrease of $0^{\mathrm{m}} \cdot 1$ during one night in 1950 and suggested that the star could be an eclipsing binary. Light variability was also reported by Feinstein \& Ferrer (1968). Mayer et al. (1992) indeed proved the eclipsing-binary nature of V1007 Sco and corrected the orbital period to 5.814 . Their light curve clearly indicates a non-zero eccentricity because of unequal spacing between the primary and secondary minimum. To avoid any confusion, we remind that the component which is eclipsed in the primary minimum is the less massive of the two. However, in accordance with the majority of published studies of V1007 Sco we shall use the usual terms primary and secondary for the stars eclipsed in the primary and secondary light minimum, respectively.

After additional $U B V$ photometry had been secured in 1993 and the Cerro Tololo RVs became available, PM was able to derive an improved value of the orbital period of $5.81600 \pm 0$ d 00003 which he communicated privately to 
Table 1. Stars in the close neighbourhood of V1007 Sco as listed in Simbad by March 2007.

\begin{tabular}{rllcll}
\hline \hline Identification & $\begin{array}{l}\alpha_{2000} \\
(\mathrm{~h}, \mathrm{~m}, \mathrm{~s})\end{array}$ & $\begin{array}{l}\delta_{2000} \\
(\mathrm{deg}, \operatorname{arcmin}, \operatorname{arcsec})\end{array}$ & $\begin{array}{c}\text { Distance from V1007 Sco } \\
(\operatorname{arcmin})\end{array}$ & $V$ & Reference \\
\hline CD-41 $11033 \mathrm{~B}$ & 165410.8 & -414936 & 0.17 & $12^{\mathrm{m}} \cdot 0$ & Cordoba Durchmusterung \\
NGC 6231*381 & 165411 & $-4149.7-$ & 0.26 & $11^{\mathrm{m}} \cdot 07$ & $\begin{array}{l}\text { Raboud et al. (1997) } \\
\text { NGC 6231*SBL 400 }\end{array} 165410.94$ \\
\hline
\end{tabular}

Table 2. RVs measured in the Cerro Tololo spectra of V1007 Sco.

\begin{tabular}{ccrrrrrrr}
\hline \hline $\begin{array}{c}\text { HJD } \\
-2400000\end{array}$ & $\begin{array}{c}\text { Pri. RV } \\
\left(\mathrm{km} \mathrm{s}^{-1}\right)\end{array}$ & $\begin{array}{r}\text { m.e. } \\
\left(\mathrm{km} \mathrm{s}^{-1}\right)\end{array}$ & $\begin{array}{r}\text { No. of } \\
\text { lines }\end{array}$ & $\begin{array}{r}\text { Sec. RV } \\
\left(\mathrm{km} \mathrm{s}^{-1}\right)\end{array}$ & $\begin{array}{r}\text { m.e. } \\
\left(\mathrm{km} \mathrm{s}^{-1}\right)\end{array}$ & $\begin{array}{r}\text { No. of } \\
\text { lines }\end{array}$ & $\begin{array}{r}\text { Ca II (K line) } \\
\left(\mathrm{km} \mathrm{s}^{-1}\right)\end{array}$ & Telescope \\
\hline 43646.681 & $-21.2^{b}$ & 4.0 & 5 & & & & -25.7 & $0.91-\mathrm{m}$ \\
43647.872 & -179.3 & 5.2 & 4 & 202.0 & 12.2 & 2 & -18.0 & $0.91-\mathrm{m}$ \\
43648.908 & -57.5 & 4.4 & 4 & 80.2 & 4.9 & 3 & -16.7 & $0.91-\mathrm{m}$ \\
43650.933 & 192.4 & 4.5 & 8 & -250.0 & 5.2 & 9 & -13.1 & $1.52-\mathrm{m}$ \\
43651.712 & $-64.9^{b}$ & 3.5 & 8 & & & & -20.4 & $1.52-\mathrm{m}$ \\
\hline
\end{tabular}

" $b$ ": RV values measured near conjunction, when the spectral lines of both components are severely blended.

Dr. D. J. Stickland. Guided by his result, Stickland et al. (1996) analysed the IUE spectra of V1007 Sco and derived a new set of orbital elements and the value of the orbital period similar to Mayer's value, $5.816083 \pm 0$ d 000019 . They confirmed that the orbit has a significant non-zero eccentricity of about $0.1-0.15$. A detailed study was carried out by Penny et al. (1999) who adopted the orbital period of Stickland et al. (1996) and derived the first solution of the light curve and all basic physical properties of the binary system. They classified the individual component spectra as O7I + O7I after a detailed analysis of the IUE spectra. They obtained component masses of 24 and $26 M_{\odot}$ and pointed out that these masses are too low if compared to evolutionary tracks of single stars. Sana et al. (2001) published another detailed study based on optical spectra from several different instruments, in which they arrived at masses of 29.6 and $29.9 M_{\odot}$, and pointed out that the stars are coming close to their limit of stability near periastron. They interpreted the phasedependent line-profile variability of $\mathrm{H} \alpha$ and He II 4686 lines as due to a strong stellar-wind interaction between the two stars.

V1007 Sco may actually be a triple or even quadruple system. In the Simbad database (March 2007), three different objects close to V1007 Sco are listed - see Table 1. Having inspected real photographs of this field, we suspect that these three objects are identical and that there is only one visual companion of V1007 Sco. The only disturbing fact is a 1-mag difference in the $V$ magnitudes derived by Raboud et al. (1997) and Sung et al. (1998), since both studies are based on well-standardized photometric measurements, and because the $V$ magnitudes of other stars observed by both teams agree quite well. Perryman \& ESA (1997) derived the separation of NGC 6231*SBL 400 from V1007 Sco to be 13 ".52 and give its magnitude as 9m.82-9m.87, i.e. some $3^{\mathrm{m}} .75$ fainter than V1007 Sco. We point out that this star was always well outside the diaphragm during our LaSilla and SAAO photoelectric observations (see below).

Mason et al. (1998) reported detection of a speckleinterferometric companion (measured separation 0.'087 on one, and 0.'048: on another occasion). According to the electronic Washington Double Star catalog ${ }^{1}$, this component was not resolved on two other occasions. According to Mason (priv. comm.) the component was unresolved during July 2001 observation with a 4-m telescope while in 2006 it was resolved

\footnotetext{
${ }^{1}$ Mason, B.D., Wycoff, G.L., Hartkopf, W.I.

http://ad.usno.navy.mil/wds
}

at 0.052 , the weak signal implying probable $\Delta m>3$ mag. Therefore, the very existence of this object requires further confirmation.

Since there are still very few reliable direct determinations of masses and radii of O-type stars, the importance of detailed studies of V1007 Sco is obvious, especially since it is also a member of the cluster NGC 6231.

\section{Observations and reductions}

\subsection{Spectroscopy}

We are able to add new RVs obtained with three different spectrographs:

1. One of us, NM, re-measured the two Cerro Tololo spectra having a linear dispersion of $39 \mathrm{~A} \mathrm{~mm}^{-1}$ and published by Levato \& Morrell (1983). She derived also three new RVs from Cerro Tololo spectra with a lower dispersion of $60 \mathrm{~A} \mathrm{~mm}^{-1}$ which were obtained at about the same time with the 0.91-m Cassegrain reflector. All these new RV measurements were obtained with the help of a Grant oscilloscopic measuring machine and are summarised in Table 2.

2. In 1992 and 1993, two of us (PM and RL) obtained 13 new CCD spectra of high $\mathrm{S} / \mathrm{N}$ using the Echelec coudé spectrograph attached to the ESO 1.52-m telescope at La Silla (dispersion $3.8 \mathrm{~A} \mathrm{~mm}^{-1}$ ). Due to some defects of the CCD detector only a few spectral lines were usable for RV measurements. The Echelec spectra show the He II 4686 line as a very broad weak emission with superimposed absorption lines from both components.

3. In 1994, 10 additional CCD spectra were secured by RL with the CAT/CES spectrograph and short camera. They have a dispersion of $2.6 \mathrm{~A} \mathrm{~mm}^{-1}$ and cover only one line per exposure, usually He I 4922, but $\mathrm{H} \alpha$ was observed as well. The initial reduction of all Echelec and CAT spectra was carried out using the MIDAS software package. The rectification and removal of cosmic spikes were carried out with the program SPEFO - see Horn et al. (1996); Škoda (1996).

We recompiled also the RVs from the literature and - whenever the original tabulation contained either dates of mid-exposures or geocentric JDs - we calculated heliocentric Julian dates (HJDs hereafter) with the help of the HEC19 computer program 
Table 3. Journal of the RV data sets. See text for identification of data sources A to I.

\begin{tabular}{cccrc}
\hline \hline $\begin{array}{c}\text { Spg. } \\
\text { No. }\end{array}$ & $\begin{array}{c}\text { Time interval } \\
\text { (HJD-2 400 000) }\end{array}$ & $\begin{array}{c}\text { No. of RVs } \\
\text { prim./sec. }\end{array}$ & $\begin{array}{r}\text { Resolution } \\
\lambda \cdot d \lambda^{-1}\end{array}$ & Source \\
\hline 1 & $26482.9-26517.2$ & $2 / 0$ & 1400 & $\mathrm{~A}$ \\
2 & $31210.9-31225.9$ & $34 / 29$ & 2600 & $\mathrm{~B}$ \\
3 & $40808.6-40815.6$ & $14 / 10$ & 2600 & $\mathrm{C}$ \\
4 & 39957.0 & $1 / 0$ & 6200 & $\mathrm{D}$ \\
4 & $43650.9-43651.7$ & $0 / 2$ & 2700 & $\mathrm{E}$ \\
5 & $44121.8-49983.0$ & $26 / 26$ & 10000 & $\mathrm{~F}$ \\
\hline 3 & $43646.7-43648.9$ & $3 / 2$ & 1750 & $\mathrm{G}$ \\
4 & $43650.9-43651.7$ & $1 / 2$ & 2700 & $\mathrm{G}$ \\
6 & $48675.8-49149.7$ & $13 / 13$ & 27000 & $\mathrm{G}$ \\
7 & $49448.7-49454.8$ & $10 / 10$ & 52000 & $\mathrm{G}$ \\
8 & $49910.7-51361.6$ & $8 / 6$ & 14000 & $\mathrm{I}$ \\
9 & $50205.9-50539.9$ & $17 / 17$ & 3700 & $\mathrm{H}$ \\
10 & $50531.9-51000.7$ & $12 / 12$ & 70000 & $\mathrm{H}$ \\
11 & $51299.8-51673.8$ & $13 / 13$ & 48000 & $\mathrm{H}$ \\
12 & $51328.7-51332.8$ & $5 / 5$ & 45000 & $\mathrm{H}$ \\
\hline
\end{tabular}

Abbreviations in column "Spg. no.":

1 Lick 0.91-m refractor, one-prism spg., $75 \mathrm{~A} \mathrm{~mm}^{-1}$; 2 McDonald, Cassegrain reflector, quartz prism spg., $40 \mathrm{~A} \mathrm{~mm}^{-1}$; 3 Cerro Tololo 0.91-m Cassegrain reflector, grating spg., dispersions $40 \mathrm{~A} \mathrm{~mm}^{-1}$ (source C) and $60 \mathrm{~A} \mathrm{~mm}^{-1}$ (source G); 4 Cerro Tololo 1.52-m reflector, coudé grating spg., $17 \mathrm{~A} \mathrm{~mm}^{-1}$ (source D) and $39 \mathrm{~A} \mathrm{~mm}^{-1}$ (sources E and G); 5 IUE Satellite high-dispersion SPW spectra; $6 \mathrm{La}$ Silla ESO 1.5-m reflector, coudé Echelec spg.; 7 La Silla ESO 1.4-m coudé auxiliary telescope (CAT) and coudé echelle spectrometer (CES) with the original short camera; 8 Complejo Astronómico El Leoncito (CASLEO) 2.1-m reflector, REOSC echelle spectrograph with a TEK $1024 \times 1024$ CCD; 9 La Silla ESO 1.5-m reflector, Boller and Chivens spg.; 10 La Silla ESO 1.4-m coudé auxiliary telescope (CAT) and coudé echelle spectrometer (CES), long and very long cameras; 11 La Silla ESO 1.5-m reflector, FEROS echelle spg.; 12 Cerro Tololo $1.5-\mathrm{m}$ reflector, bench-mounted echelle spg.

written by $\mathrm{PH}^{2}$. Available data sets are summarized in Table 3. A few comments are appropriate:

Source A: Hayford (1932) does not mention line doubling in her low-dispersion $\left(75 \mathrm{~A} \mathrm{~mm}^{-1}\right.$ ) spectra and these velocities cannot be reconciled with the binary orbit. If one assigns these RVs to both binary components and calculates a trial orbital solution along with other data sets, one gets a reasonable systemic velocity of $-29 \mathrm{~km} \mathrm{~s}^{-1}$, however. We do not use this early data set in the orbital solutions presented here.

Source B: The tabulation by Struve (1944) for V1007 Sco (his Table 2, Sect. 10 on page 195) is a bit confusing: while the plus or minus sign is used for each RV elsewhere in the table, here he omitted the minus sign for all but the first and the last $\mathrm{RV}$ in the first column with the velocities. Note also that the RVs given in the two consecutive columns of Struve's Table 2 refer alternatively to the primary and secondary, even for the period of 3.10 days preferred by Struve (cf. his Fig. 2). Our final identification of RVs of both components is shown in Fig. 1. RVs from the spectra taken on May 3, 1944 at 7:25 UT, and May 15, 1944 at 9:41 UT, denoted as poor by Struve (1944) were omitted in our analysis.

Source C: Hill et al. (1974) interchange the primary and secondary components compared to our nomenclature. The Julian dates which they give for a selection of Struve's spectra they used, denoted $\mathrm{JD}_{\odot}$, are not heliocentric in fact.

Source D: A single RV published by Conti et al. (1977).

2 The program with sample types of input data is available to interested colleagues at ftp://astro.troja.mff.cuni.cz/hec/HEC19

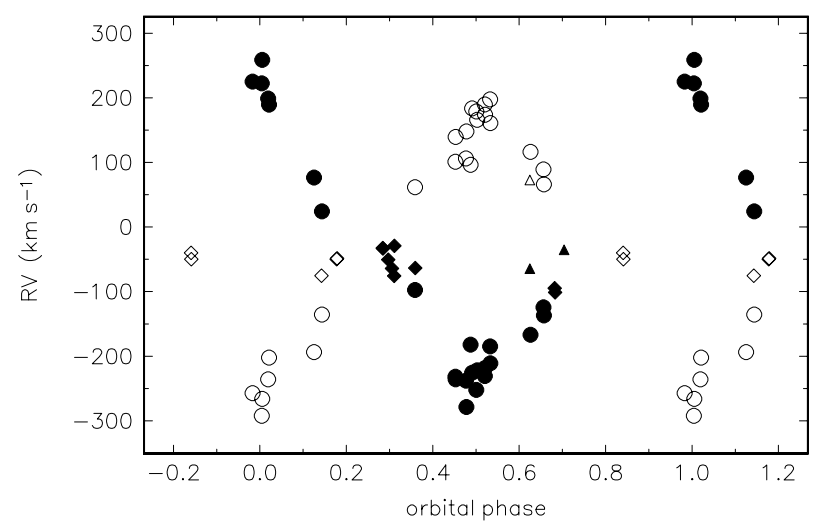

Fig. 1. A phase plot of RVs from Struve (1944) for the period of 5.8160 and using HJD 2431216.0 as phase zero. The RVs identified by us as belonging to the primary are shown by filled symbols, those of the secondary by empty symbols. RVs from the spectra where both components were resolved are shown by large circles, those from the spectra where only one RV was measured by diamonds and the three RVs which Struve denoted as "poor" by triangles.

Source E: We found that the correct HJDs of the spectra given in Levato \& Morrell (1983) are HJD 2443650.9331 and ...651.7123. As mentioned above, these spectra were remeasured by NM. Therefore, we do not use the original RVs from this source.

Source F: RVs from IUE spectra published by Penny et al. (1999). They cover two separate time intervals, JD 2444 121.8-2444 460.0, and JD 2448 864.0-2449 983.0.

Source G: This paper; spectra from three sources described above.

Source H: Sana et al. (2001) published RVs of He I 4471 and mean RVs, but in both cases already with the mean RV of the respective subset subtracted. We use a homogeneous set of He I 4471 RVs in the original heliocentric RV scale, which were kindly provided to us by Dr. H. Sana.

Source I: García \& Mermilliod (2001) published and analysed $8 \mathrm{RVs}$ and arrived at a period of $5 \mathrm{~d} .75$. We first could not reconcile these RVs with other available data. Upon our inquiry, Dr. J. C. Mermilliod kindly informed us that all HJDs for the last 5 spectra (HJD larger than 2450000 ), published in their Table 2, must be decreased by one day to obtain the correct HJDs. After this correction, after interchanging the primary and secondary $\mathrm{RV}$ s for the first and third spectrum, and neglecting the RV of $(-147.7 \pm 17.1) \mathrm{km} \mathrm{s}^{-1}$ for the secondary at HJD 2450593.565 because of its large error, we were able to reconcile these RVs with the 5.816 period.

For convenience of future investigators, we present all RVs, both the recompiled and the new ones together with the corresponding heliocentric Julian dates (HJDs) in Table $4^{3}$.

\subsection{Photometry}

The photometry of V1007 Sco is still rather scarce and new observations are very desirable. The data at our disposal consist of the following three sets of photoelectric observations and one set of far-UV magnitudes:

1. $U B V$ observations obtained by Mayer et al. (1992) plus additional $U B V$ observations secured by RL. The stars HD 152147 and HD 152314 served as the comparison and

3 Table 4 is only available electronically. 
check, respectively. The all-sky $U B V$ magnitudes of the comparison star HD 152147

$V=7^{\mathrm{m}} .265 \pm 0^{\mathrm{m}} \cdot 008$,

$B=7^{\mathrm{m}} .672 \pm 0^{\mathrm{m}} .010$, and

$U=7^{\mathrm{m}} .061 \pm 0^{\mathrm{m}} .011$,

which were added to the magnitude differences var-comp, were derived by us from a careful reduction of all observations including several standards using the HEC22 program - see Harmanec et al. (1994). For the check star HD 152314 the corresponding values read as

$V=7^{\mathrm{m}} .822 \pm 0^{\mathrm{m}} .025$,

$B=8^{\mathrm{m}} .037 \pm 0.024$, and

$U=7^{\mathrm{m}} \cdot 312 \pm 0^{\mathrm{m}} \cdot 017$.

Very regrettably, neither of the comparisons but only V1007 Sco itself was observed by Hipparcos. These La Silla $U B V$ observations of V1007 Sco and other early-type variables will be published in detail in a separate study (Mayer, in preparation).

HD 152314 has been suspected to be a variable and is listed as NVS 8027. This star as well as HD 152147 have been repeatedly observed in the yellow passband in the ASAS-3 project (Pojmanski 2002). There is no indication of variability of HD 152314, the rms of the measurements between JD 2453000 and 2453200 being smaller than $0^{\mathrm{m}} 01$. The scatter of the measurements of HD 152147 is somewhat higher, probably due to the fact that this star is brighter and therefore closer to the saturation limit of the instrument (ASAS-3 data for V1007 Sco being totally unusable). The mean ASAS-3 magnitudes of HD 152147 and HD 152314 amount to $7^{\mathrm{m}} .24$ and $7^{\mathrm{m}} .76$ in $V$, respectively.

2. The Hipparcos $H_{p}$ magnitudes, transformed into Johnson $V$ using the transformation formula given by Harmanec (1998).

3. Ten $V$ observations from Strömgren photometry published by Manfroid et al. (1994).

4. $76 U B V$ observations were secured by $\mathrm{MW}$ with the $0.5-\mathrm{m}$ reflector and a photolectric photometer of the South African Astronomical Observatory during 10 nights in 2004. Also these observations were reduced with the HEC22 program and transformed to the standard system.

5. Fluxes from the IUE spectra of the star, which were already used for a RV determination by Stickland et al. (1996), were integrated over two wavelength intervals relatively free of lines, $1600-1948 \AA$ and $1265-1375 \AA$ to obtain two far-UV magnitudes $m_{1774}$ and $m_{1320}$, which were also included in the light-curve solutions.

All individual photometric observations are provided in detail in Table $6^{4}$.

\section{RV measurements based on La Silla spectra}

Determination of reliable RVs of hot, rapidly rotating stars with some lines affected by emission from the circumstellar environment always represents a challenging task. A specific problem is the selection of spectral lines suitable for RV measurements, basically free from emission and blends. For V1007 Sco, this problem was for the first time carefully addressed by Sana et al. (2001). They first derived different "systemic velocities" for different spectral lines and then applied appropriate RV shifts to individual lines before deriving the mean RV from them. They

\footnotetext{
4 Table 6 is only available electronically.
}

Table 5. Journal of photoelectric observations.

\begin{tabular}{ccrrl}
\hline \hline $\begin{array}{c}\text { Dataset } \\
\text { No. }\end{array}$ & $\begin{array}{c}\text { Epoch } \\
\text { HJD-2400 } 000\end{array}$ & $\begin{array}{r}\text { No. of } \\
\text { obs. }\end{array}$ & $\begin{array}{r}\text { No. of } \\
\text { nights }\end{array}$ & $\begin{array}{l}\text { Passbands } \\
\text { used }\end{array}$ \\
\hline 1 & $47919-48829$ & 47 & 34 & $V$ from $H_{p}$ \\
2 & $48429-48537$ & 10 & 10 & $u v b y$ \\
3 & $48682-49460$ & 250 & 8 & $U B V$ \\
4 & $53118-53129$ & 76 & 10 & $U B V$ \\
5 & $49817-49983$ & 16 & 14 & $m_{1774} \& m_{1320}$ \\
\hline
\end{tabular}

Datasets:

1 Perryman \& ESA (1997); 2 Manfroid et al. (1994); 3 Mayer et al. (1992) and this paper; 4 this paper, $U B V$ photometry with the $0.5-\mathrm{m}$ reflector of the South African Astronomical Observatory (SAAO); 5 Fluxes from IUE spectra were integrated over the regions

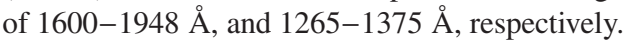

argued that the different RV zero points for different lines indicate different outward velocites in the stellar atmospheres. Since they obtained basically the same systemic velocities for each given ion for both binary components, this would imply that the same outward motion is observed in the atmosheres of both stars. We believe that there is a simpler explanation: an unrecognized line blending. Carrying out some tests with synthetic spectra, we indeed found that many lines, including the Balmer lines, are asymmetric and their effective rest wavelengths vary with $T_{\text {eff }}$, $\log g$ and $v \sin i$. Any future investigation of a possibly real variability of the systemic velocity of V1007 Sco should be based on systematic observations of particular, carefully selected spectral lines free from emission contribution. Perhaps the most objective way of deriving the true systemic velocity would be a comparison of disentagled spectra of both binary components with the best-fitting synthetic spectrum.

For the purpose of our study, we first derived RVs of individual lines from the LaSilla electronic spectra (spectrographs Nos. 6 and 7 of Table 3) by two independent methods: (1) fitting the observed line profiles with two Gaussian profiles, and (2) comparing the direct and flipped profile interactively by use of the SPEFO program. It turned out that the latter technique is more affected by strong line blending and inevitably returns RVs of both binary components with smaller than true velocity separation. We therefore finally adopted the RVs based on the fitting of Gaussian profiles. These are listed in detail in Table 7. Then we proceeded similarly as Sana et al. (2001): Using the FOTEL program, we derived a trial orbital solution, allowing RVs of individual lines to have different "systemic" velocities, separately for spectrographs 6 and 7. These mean RV were then subtracted from the measured RVs individually for each ion and spectrograph. These final mean RVs (decreased for individual systemic velocities) are listed in Table 4. Note that this seemingly complicated procedure is particularly important for our Echelec and CAT spectra which contain different lines in different spectra. Deriving the mean RV value for each spectrum without appropriate shifts would inevitably decrease the resulting RV amplitude. We do not expect that our RV measurements are significantly affected by possible weak emission in the spectral lines. Since the emission in $\mathrm{H} \alpha$ is weak, we expect that the emission in $\mathrm{H} \beta$ must be negligible. Also the He I 4713 and 4922 lines are rarely seen in emission. There is a weak and a very broad emission in He II 4686 but its effect could be avoided during the fit of the Gaussian profiles. 
Table 7. RVs (in $\mathrm{km} \mathrm{s}^{-1}$ ) of components 1 and 2 of V1007 Sco measured via a fit of two Gaussian profiles in the La Silla Echelec and CAT CCD spectrograms and tabulated individually for various stronger lines.

\begin{tabular}{crrrrrrrr}
\hline \hline HJD & \multicolumn{2}{c}{ He II 4686 } & \multicolumn{2}{c}{ He I 4713 } & \multicolumn{3}{c}{$\mathrm{H} \beta$} & \multicolumn{2}{c}{ He I4922 } \\
-2400000 & prim. & sec. & prim. & sec. & prim. & s & prim. & sec. \\
\hline Echelec & & & & & & & & \\
\hline 48675.8453 & 223.8 & -209.0 & - & - & 226.7 & -230.9 & - & - \\
48677.8524 & -193.6 & 174.9 & -144.6 & 163.3 & -206.2 & 158.3 & - & - \\
48678.8407 & -177.0 & 181.3 & - & - & -200.0 & 184.8 & - & - \\
49023.8355 & - & - & - & - & - & - & 122.3 & -151.2 \\
49027.8809 & -192.3 & 155.1 & -182.1 & 176.6 & -169.8 & 171.9 & -170.1 & 191.2 \\
49029.8769 & 174.3 & -141.8 & 133.4 & -151.0 & 156.4 & -120.8 & 152.1 & -172.9 \\
49146.7069 & - & - & - & - & 210.7 & -203.7 & 231.9 & -197.5 \\
49148.7201 & - & - & - & - & - & - & -142.7 & 140.6 \\
49149.6896 & - & - & - & - & -200.0 & 218.8 & - & - \\
\hline CAT/CES & & - & - & - & & & & \\
\hline 49448.7291 & - & - & - & - & - & - & 169.8 & -184.1 \\
49448.8325 & - & - & - & - & - & - & 169.8 & -165.9 \\
49451.6932 & - & - & - & - & - & - & -198.7 & 206.3 \\
49451.7147 & - & - & - & - & - & - & -198.7 & 206.3 \\
49451.7661 & - & - & - & - & - & - & -206.1 & 198.4 \\
49454.6774 & - & - & - & - & - & - & 171.6 & -170.4 \\
49454.7483 & 164.7 & -168.0 & - & - & - & - & - & - \\
49454.7712 & 177.5 & -165.4 & - & - & - & - & - & - \\
\hline
\end{tabular}

\section{Orbital and light curve solutions}

A consistent solution of the eclipse light curve and the radial velocity curve poses a difficult problem in the case of an eccentric orbit. The non-circular motion of the stars and the apsidal line rotation continuously change the orbital geometry. Both photometric and RV measurements cover an extended time interval during which these changing conditions largely influence the shape of light and RV curves and must be properly accounted for.

The initial analyses presented here were carried out with the help of the computer program FOTEL (Hadrava 1990, 2004a). Both, orbital and light curve solutions can be obtained with FOTEL, either separately or simultaneously, and it is also possible to include the rate of apsidal advance among the elements of the solution. Other convenient features of FOTEL are that it allows calculation of individual zero points for individual data sets and also a simultaneous solution for observations in several different bandpasses. FOTEL models the shape of the stars as triaxial ellipsoids and experience shows that this gives acceptable results even for binaries close to filling their respective Roche lobes.

To arrive at the most probable orbital elements when combining spectra of widely different dispersions and resolutions, or photometry from different instruments, some reasonable weighting scheme should be applied. In this study we always first calculated either an orbital or light curve solution based on all available data sets using equal weights of 1 for all data and derived the rms per one observation for each data set separately. The global weight of each individual data set was then made inversely proportial to the square of the respective rms error. The weights were normalized in such a way that weight 1 corresponds to the mean rms of the whole set of all RVs or photometry.

Subsequently we proceeded in the following steps.

\subsection{Solutions of RV subsets localized in time}

To find an initial reasonable value of the rate of apsidal advance, we first calculated solutions corresponding to limited intervals of time, keeping the orbital period fixed at the value of 5.816 and assigning weight 1 to all RVs. These tentative solutions are
Table 8. Tentative FOTEL orbital solutions for several subsets of RV data.

\begin{tabular}{lrrr}
\hline \hline Element & spg. 2 & spg. 3, 4, \&5 & spg. 5-12 \\
\hline Epoch & $31211-31225$ & $39957-44460$ & $48676-51674$ \\
$T_{\text {periastr. }}$ & 31215.50 & 42638.97 & 50602.101 \\
& \pm 0.28 & \pm 0.27 & \pm 0.052 \\
$\omega$ & $0.132 \pm 0.029$ & $0.107 \pm 0.026$ & $0.122 \pm 0.006$ \\
$K_{1}\left(\mathrm{~km} \mathrm{~s}^{-1}\right)$ & $-27 \pm 17$ & $12 \pm 17$ & $78.4 \pm 3.3$ \\
$K_{1} / K_{2}$ & $220 \pm 18$ & $198 \pm 18$ & $213.8 \pm 1.9$ \\
$\gamma\left(\mathrm{km} \mathrm{s}^{-1}\right)$ & $1.055 \pm 0.056$ & $1.004 \pm 0.057$ & $1.015 \pm 0.013$ \\
& $-29.9 \pm 4.7$ & $-24.9 \pm 5.4$ & $-28.4 \pm 1.8$ \\
& & $-22.6 \pm 8.7$ & $-0.1 \pm 3.9$ \\
& & $-20.1 \pm 5.2$ & $0.2 \pm 2.9$ \\
& & & $-24.9 \pm 2.9$ \\
& & & $-31.6 \pm 2.5$ \\
& & & $-28.7 \pm 2.3$ \\
No. of RVs & $23 / 23$ & & $-28.0 \pm 2.7$ \\
rms (km s$\left.{ }^{-1}\right)$ & 29.9 & $20 / 19$ & $-40.0 \pm 3.9$ \\
& & 22.6 & $85 / 84$ \\
& & & 12.5 \\
\hline
\end{tabular}

The orbital period was kept fixed at $P=5.8160$. Only RVs from spectra where both components were resolved were used. All epochs are in HJD-2 400 000. Quoted rms errors are errors of 1 observation of unit weight.

collected in Table 8. It is immediately seen that the value of the periastron passage is indeed increasing with time. Figure 2 shows the secular change of $\omega$ with time and leaves little doubt about the presence of a measurable apsidal motion of V1007 Sco.

We found also that there is no significant difference in the systemic velocities of the primary and secondary and we kept a common systemic velocity for each data set in the solutions. The differences in systemic velocities from various spectrographs probably do not indicate real changes but the effect of line blending and the fact that different authors used different selections of spectral lines. Note also that RVs for spectrographs 6 and 7 have the systemic velocities already subtracted and that is why they give $\gamma$ near zero. 
Table 9. A comparison of FOTEL orbital and light curve solutions for all resolved RVs and all available photometric data with known times of observations.

\begin{tabular}{llll}
\hline \hline Element & $\begin{array}{l}\text { Resolved RVs } \\
\text { (all data) }\end{array}$ & $\begin{array}{l}\text { Resolved RVs } \\
\text { (without source B) }\end{array}$ & $\begin{array}{l}\text { Photometry } \\
\text { (all datasets) }\end{array}$ \\
\hline$P_{\text {sider. }}(\mathrm{d})$ & 5.816036 & 5.81600 & 5.816032 \\
$P_{\text {anomal. }}(\mathrm{d})$ & $5.816586 \pm 0.000058$ & $5.81679 \pm 0.00011$ & $5.816954 \pm 0.000049$ \\
$T_{\text {periastr. }}$ & $48502.334 \pm 0.047$ & $48502.288 \pm 0.052$ & $48502.351 \pm 0.015$ \\
$T_{\text {prim.ecl. }}$ & 48502.643 & 48502.618 & 48502.633 \\
$T_{\text {sec.ecl. }}$ & 48499.870 & 48499.927 & 48499.931 \\
$e$ & $0.1227 \pm 0.0056$ & $0.1226 \pm 0.0058$ & $0.1279 \pm 0.0036$ \\
$\omega($ deg.) & $66.9 \pm 3.0$ & $63.5 \pm 3.3$ & $66.9 \pm 1.0$ \\
$\dot{\omega}\left(\right.$ deg. $\left.\mathrm{d}^{-1}\right)$ & $0.00584 \pm 0.00064$ & $0.0084 \pm 0.0013$ & $0.00981 \pm 0.00058$ \\
$K_{1}\left(\mathrm{~km} \mathrm{~s} \mathrm{~s}^{-1}\right)$ & $210.9 \pm 1.9$ & $210.7 \pm 1.9$ & - \\
$K_{1} / K_{2}$ & $1.018 \pm 0.012$ & $1.015 \pm 0.012$ & - \\
$r_{1}$ & - & - & $0.284 \pm 0.014$ \\
$r_{2}$ & - & - & $0.343 \pm 0.004$ \\
$i($ deg.) & - & - & $65.67 \pm 0.32$ \\
rms (km s & 14.09 & 13.17 & - \\
rms $\left(\mathrm{mag}^{-1}\right)$ & - & 208 & 0.0111 \\
No. of $\mathrm{RV}_{1} \& \mathrm{RV}$ & 254 & - & - \\
No. of photom. obs. & - & & 1099 \\
\hline
\end{tabular}

The rate of apsidal motion was included among the elements of the solutions. All epochs are in HJD-2 400000.

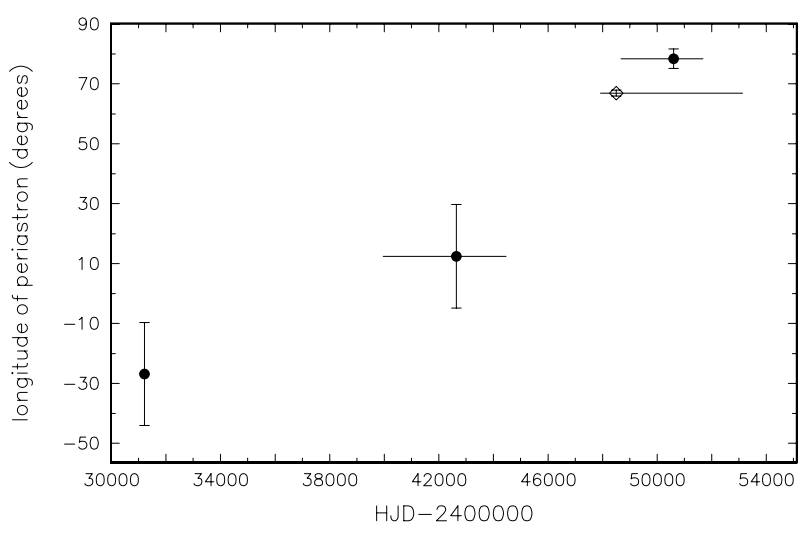

Fig. 2. A plot of the values of the longitude of periastron, derived from the RV subsets of Table 8 (filled circles) and from the photometric solution of Table 9 (empty diamond), vs. time. The error bars show the rms errors of individual determinations while the horizontal lines indicate the time intervals covered by the respective data subsets on which the locally derived values of $\omega$ are based.

\subsection{Further tentative solutions}

Then we used all available RVs for which primary and secondary components were resolved and derived a combined solution, now allowing also for the apsidal advance and using the appropriate weights for individual data sets as explained above. This solution is given in Col. 2 of Table 9 and confirms the reality of the apsidal motion in the system.

The next step was to calculate another solution using all photoelectric observations. Here, the problem is with the reported faint companions to V1007 Sco. No magnitude estimate is given for the - still putative - closer companion, neither by Mason et al. (1998) nor in the Washington catalog. It is clear that the Hipparcos photometry is free from the light of the more distant companion at 13 '.52 and the same is true for our photometry as well. Given this, our approach was the following: we neglected the possible presence of the closer component. To set an upper limit on the orbital inclination, we calculated a trial photometric solution in such a way that we assumed that there is a tertiary which is $3^{\mathrm{m}} .7$ fainter than the total brightness of the triple system in all five passbands. Compared to a solution where only the binary is considered this leads to an increase of the orbital inclination by 0.4 . On the other hand, having no information on the effective temperature of the tertiary, including any such body in the solution complicates the correct determination of the luminosity ratio of the binary components. We, therefore, decided to restrict ourselves to the binary light curve solutions only. One should be aware that should the existence of the closer companion of V1007 Sco be confirmed and should this component contribute significantly to the total luminosity of the system, our solutions would define only a lower limit of the orbital inclination. We note, however, that even if one adopts a magnitude difference of 2 mag as Penny et al. (1999) did, the light curve solution gives an inclination of $67^{\circ} .5$, not $72^{\circ} .0$ like obtained as the best fit by Penny et al. (1999). A probable explanation lies in the fact that Penny et al. (1999) only simulated an eccentric orbit in their solution and used a program which does not treat apsidal motion (being actually unaware of its presence at the time of their analysis).

Our solution for all (weighted) photometric data is also presented in Table 9.

At this point we are facing a problem. While most of the elements common to the orbital and light curve solutions agree quite well, there is a large difference in the rate of the apsidal advance between the two solutions. We derived another orbital solution, using all RVs with the exception of the first data set obtained by Struve (1944). This solution is presented in the third column of Table 9. This time, the mutual agreement between the orbital and light curve solutions is very satisfactory. For the moment, we shall therefore proceed without Struve's RVs, but we consider this disagreement a disturbing one since there is no a priori reason to doubt about this early data set (as discussed in detail above). Therefore systematic observations of the binary should clearly be continued.

Using all electronic spectra from ESO (Echelec and CAT), we applied also the program KOREL (Hadrava 1995, 1997, 2004b, 2005) and derived a joint KOREL solution using the following four spectral lines: He II 4686, He I 4713, He I 4922 and $\mathrm{H} \beta$, allowing for the variable line strength of individual 
spectra. The result confirmed the orbital solutions from FOTEL. However, because of the scarcity and heterogenity of the spectra available to us, we used the disentangled profiles for a better estimate of the projected rotational velocities of both components see below.

\subsection{Final PHOEBE solution}

Our objective was to carry out a simultaneous solution of all available RV measurements and photometric data from the various sources. To this goal, we used the computer program PHOEBE (PHysics Of Eclipsing BinariEs) developed by Prša \& Zwitter (2005), which is a user-friendly implementation of the Wilson-Devinney (hereafter WD) code (Wilson \& Devinney 1971). The WD light curve synthesis is based on the Roche geometry, which ensures a realistic representation of the changing shape of the stars at all phases of the eccentric orbit. The analytic part of the program which improves the initial values of the physical elements of the solution via differential corrections is specifically designed to solve the RV and light curves simultaneously.

The synthetic PHOEBE light curves can be convolved with a built-in set of transmission curves of Johnson $U B V$ and Strömgren $u v b y$ filters. Linear limb-darkening coefficients were automatically interpolated by PHOEBE from the tables of van Hamme (1993).

An important parameter in the analysis of any early-type binary is the temperature of its primary component. A recent study by Heap et al. (2006) suggests a temperature of $35000 \mathrm{~K}$ for giants of $\mathrm{O} 7$ to $\mathrm{O} 8$ type in the SMC, while stars with solar abundance are about 1000-2000 K cooler. In the case of V1007 Sco similar values are also indicated by the ratio of equivalent widths of the He I 4542 and 4471 lines - giving a somewhat lower temperature for the primary component as would follow from EW ratios by Sana et al. (2001). It is also known that the secondary temperature is about $1000 \mathrm{~K}$ higher than that of the primary (Penny et al. 1999). Therefore, we chose $34000 \mathrm{~K}$ for the primary and adjusted the secondary temperature in our light curve solution.

First, we derived the improved values of inclination, eccentricity and time of periastron using only the light curves (which span some 4500 days). Subsequently, the spectroscopic observations (spanning a much longer time interval of more than 50 years) were considered to derive the final values of the orbital period and the rate of apsidal advance as well. During this process a simultaneous analysis of all available light and RV curves was not appropriate because of the large number of free parameters. Therefore separate data sets each covering relatively narrow time intervals were used for the determination of a limited number of selected parameters, which then again served as input for the next iteration with another subset of parameters adapted to a new data set and so on.

After a satisfactory fit of all available data had been achieved by the separate analysis of light and RV curves, a final simultaneous solution of all data was obtained. To avoid any subjective influence on the fitting process we employed an automatic iteration scheme known as the "bootstrap algorithm" using the PHOEBE scripter module (pre-release of version 0.30), which works as follows: for a light curve containing $n$ data points, the following procedure is repeated $n$ times: a data point is randomly drawn from all light curve points and stored in a new file. It is not removed from the original file; rather, the same point could be drawn multiple times. Thus the new "information reduced" light curve contains anywhere between 1 and $n$ data points. The
Table 10. Absolute parameters of V1007 Sco .

\begin{tabular}{lcc}
\hline \hline Parameter & Solution 1 & Solution 2 \\
\hline$P_{\text {sider. }}($ day $)$ & $5.8160658 \pm 0.0000009$ & fixed \\
$T_{\text {peri. }}$ & $2448502.380 \pm 0.001$ & fixed \\
$T_{\text {prim. min. }}$ & $2448502.637 \pm 0.001$ & $2448502.636 \pm 0.001$ \\
$e$ & $0.1224 \pm 0.0011$ & $0.12805 \pm 0.00079$ \\
$\omega($ deg $)$ & $69.67 \pm 0.44$ & $69.36 \pm 0.42$ \\
$\dot{\omega}\left(\right.$ deg. $\left.\mathrm{d}^{-1}\right)$ & $0.00884 \pm 0.00012$ & fixed \\
$i($ deg $)$ & $67.43 \pm 0.22$ & $67.20 \pm 0.22$ \\
$K_{1}\left(\mathrm{~km} \mathrm{~s}^{-1}\right)$ & $211.7 \pm 2.3$ & $216.8 \pm 1.7$ \\
$K_{2}\left(\mathrm{~km} \mathrm{~s}^{-1}\right)$ & $206.9 \pm 2.2$ & $212.6 \pm 1.6$ \\
$\gamma\left(\mathrm{km} \mathrm{s}^{-1}\right)$ & $-29.27 \pm 0.04$ & see text \\
$r_{1}$ & $0.280 \pm 0.014$ & $0.298 \pm 0.014$ \\
$r_{2}$ & $0.309 \pm 0.007$ & $0.289 \pm 0.007$ \\
$A\left(R_{\odot}\right)$ & $51.70 \pm 0.08$ & $53.08 \pm 0.39$ \\
$T_{\text {eff.1 }}$ & $34000 \mathrm{~K}$ adopted & $34000 \mathrm{~K}$ adopted \\
$T_{\text {eff. } .2}$ & $34350 \mathrm{~K}$ & $34330 \mathrm{~K}$ \\
$L_{2} /\left(L_{1}+L_{2}\right)(V)$ & $0.56 \pm 0.01$ & $0.493 \pm 0.010$ \\
$M_{1}\left(M_{\odot}\right)$ & $27.24 \pm 0.15$ & $29.46 \pm 0.39$ \\
$M_{2}\left(M_{\odot}\right)$ & $27.88 \pm 0.16$ & $30.05 \pm 0.39$ \\
$R_{1}\left(R_{\odot}\right)$ & $14.47 \pm 0.71$ & $15.84 \pm 0.71$ \\
$R_{2}\left(R_{\odot}\right)$ & $15.99 \pm 0.47$ & $15.32 \pm 0.47$ \\
$M_{1, \text { bol }}$ & $-8.76 \pm 0.10$ & $-8.96 \pm 0.10$ \\
$M_{2, \text { bol }}$ & $-9.05 \pm 0.06$ & $-8.83 \pm 0.06$ \\
$M_{1, \mathrm{v}}$ & $-5.52 \pm 0.10$ & $-5.72 \pm 0.10$ \\
$M_{2, \mathrm{v}}$ & $-5.78 \pm 0.06$ & $-5.64 \pm 0.06$ \\
\hline & &
\end{tabular}

whole procedure is repeated a large number of times to produce a set of randomly modified light curves from the original data. Afterwards, the resulting light curves are subjected to exactly the same fitting scheme as the original data set was. Each analysis will yield a slightly different solution parameter set. From these, mean values along with associated standard errors are computed. The mean value of each parameter is adopted as its final value, while the bootstrap-derived errors can serve as realistic estimates for the quality of the parameter determination. Detailed information about the bootstrap method can be found in Efron (1979) and Efron \& Tibshirani (1979). The relative weights of light curve and radial velocity data were chosen such that both data contributed approximately equally to the final errors but the weighting scheme also suppressed the data sets with large rms errors. The results of this procedure are tabulated as solution 1 in Table 10.

In is known that low-resolution spectra can decrease the semiamplitudes of the RV curves. Therefore, to obtain the final solution representative for masses and radii, we fixed the period and apsidal advance from solution 1 and derived another solution, solution 2 in Table 10, based again on all photometric observations but on RVs from only those spectra having resolution 20000 or higher. Individual systemic velocities were subtracted from the RV data (that is why we do not give the value of $\gamma$ for this solution) and the weights of individual data sets were made inversely proportional to the square of the respective rms errors. The errors of parameters in solution 2 are also the above described bootstrap errors. They should be a more realistic than any other formal fitting errors ${ }^{5}$.

The theoretical light curves resulting from solution 2 are compared with observations in Figs. 3-5. Due to the changing

\footnotetext{
5 We tried also to derive the period and the apsidal advance from data on which solution 2 is based. It gave $P_{\text {sider. }}=5.816036(7)$ and $\dot{\omega}=0.0115$ (4) deg. per day. It will be shown below that this shorter apsidal period agrees with the evolutionary model better than that from solution 1 but since the time interval covered is rather short, we prefer to use the values of solution 1 for the moment.
} 


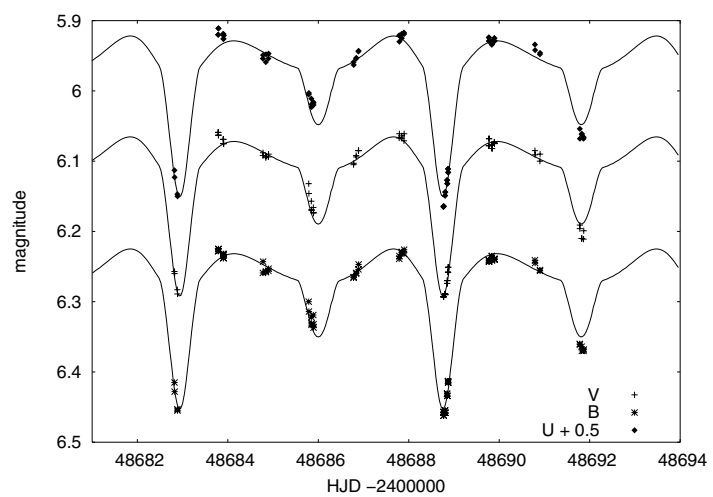

Fig. 3. Light curve constructed for data obtained during 1992.

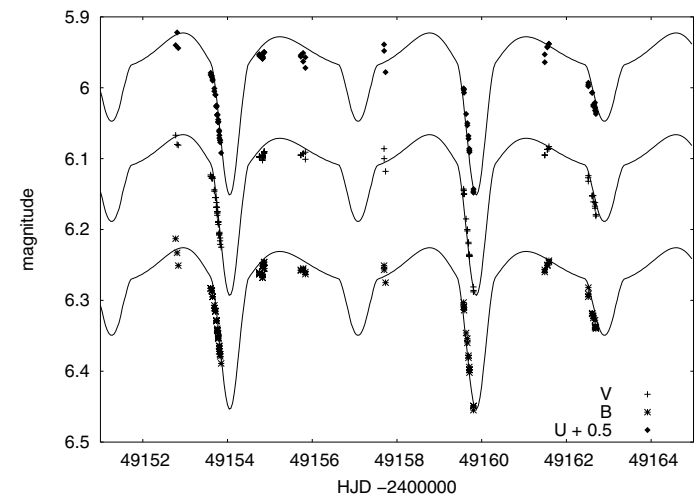

Fig. 4. Light curve constructed for data obtained during 1993.

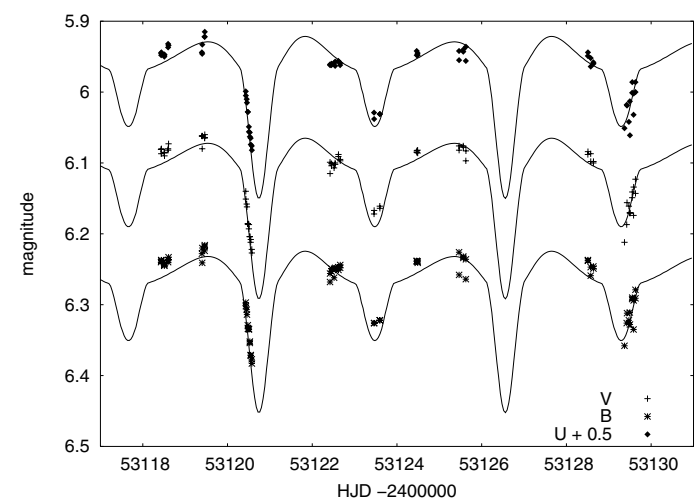

Fig. 5. Light curve constructed for data obtained during 2004.

periastron longitude, the curves are shown for limited time intervals during which the apsidal line rotation causes no recognizable effect on the light curve shape. For the same reason, the RV curves are shown in Fig. 7 only for our CAT/CES data and data by Sana et al. (2001) and García \& Mermilliod (2001) i.e. for JDs larger than 2449448 . It should be noted that the theoretical curves corresponding to solutions 1 and 2 coincide so closely that their differences would not be perceptible in figures.

Theoretical curves were calculated for the UV wavelengths 1320 and $1774 \AA$, too, and are shown together with the observed magnitudes (see Sect. 2.2, set 5) in Fig. 6.

\section{Distance, rotation and apsidal line rotation}

Using the data of Table 10, the absolute magnitude $M_{V}$ of the binary is -6.43 (solution 2). With $V=6.08$ in maximum and $E_{B-V}=0.47$ (absorption 1.46 ), $V_{0}$ is 4.62 ; then the distance

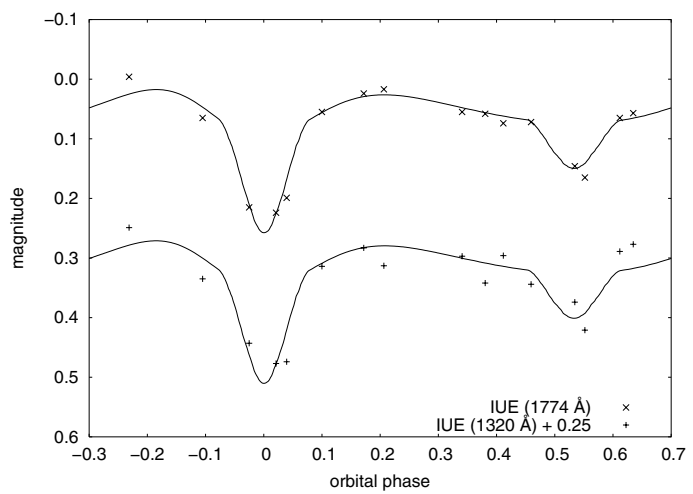

Fig. 6. Light curves constructed for the IUE continua with central wavelengths 1320 and $1774 \AA$.

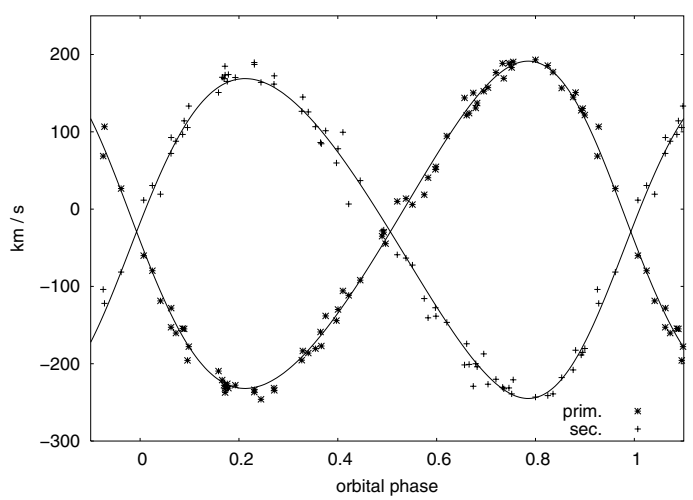

Fig. 7. Radial velocity curve constructed for data with JD larger than 2449 448. Phase zero corresponds to the primary minimum.

Table 11. Distance of NGC 6231.

\begin{tabular}{ll}
\hline \hline Source & Distance modulus \\
\hline Balona \& Laney (1995) & $11.08 \pm 0.05$ \\
Raboud et al. (1997) & $11.2 \pm 0.4$ \\
Baume et al. (1999) & 11.49 \\
Sana et al. (2005) & $11.07 \pm 0.04$ \\
\hline
\end{tabular}

modulus of V1007 Sco is 11.05 and the distance 1624 pc. These values were obtained using the solar temperature of $5777 \mathrm{~K}$, and solar $M_{V}$ and absorption coefficient 3.1 according to Allen's Astrophysical Quantities (1999); bolometric corrections were taken from Lanz \& Hubeny (2003).

The distance to NGC 6231 had already been determined many times; some recent values are listed in Table 11 . The distance modulus was derived also for the eclipsing binary V1034 Sco by Bouzid \& Sterken (2004) as $10.73 \pm 0.02$ and by Sana et al. (2005) as $10.92 \pm 0.16$. All these distances were obtained under different assumptions about reddening, temperature scales and main-sequence traces, which can account for the scatter in the results.

Our distance of V1007 Sco agrees quite well with those distance values of NGC 6231 for which their authors claim the smallest errors. The distance is of course connected with the often discussed problem of the discrepancy between the evolutionary and observed masses. V1007 Sco holds a record in the discrepancy between these masses in Hilditch's list (Hilditch 2004). The positions of the components of V1007 Sco in the HRD are compared with the models by Claret (2004) in Fig. 8.

The reason for the mismatch between observed and theoretical masses might be the rotation of the components. However, it 


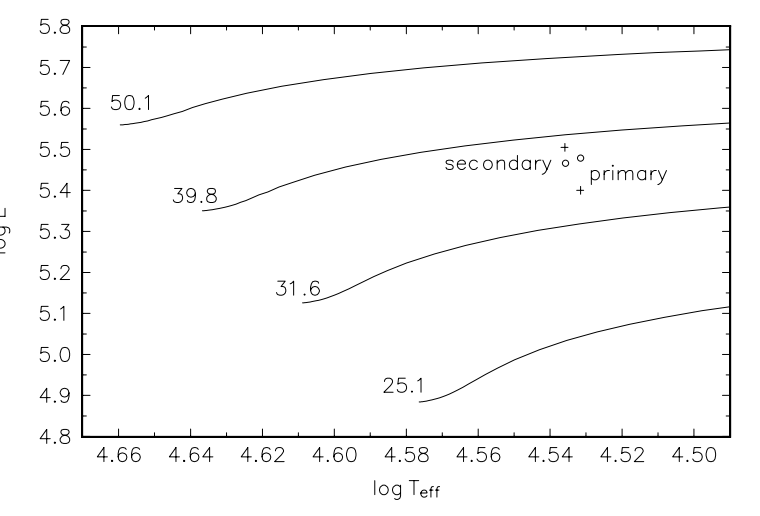

Fig. 8. Comparison of the evolutionary tracks computed by Claret (2004) with the observed parameters of the components. The numbers give ZAMS masses of the models. Crosses and open circles denote results of solutions 1 and 2 of Table 10, respectively.

appears that in this case the effect should be smaller than 0.1 in the logarithm of luminosity, as judged from Fig. 7 in Meynet \& Maeder (2000). The rotational velocity was determined in three papers, always using IUE spectra and the CCF method. Stickland et al. (1996) gave $v$ sin $i$ as $190 \mathrm{~km} \mathrm{~s}^{-1}$ for both components, Penny et al. (1999) 172 and $182 \mathrm{~km} \mathrm{~s}^{-1}$ for the primary and secondary components, and Howarth et al. (1997) gave 159 and $165 \mathrm{~km} \mathrm{~s}^{-1}$. We determined the velocities using profiles of the line 4922 as they resulted from the application of KOREL. We compared these profiles with the rotationally widened synthetic spectrum (SYNSPEC) and found that the best fit is obtained for a considerably lower $v \sin i: 135 \pm 5 \mathrm{~km} \mathrm{~s}^{-1}$ for both components. These smaller values agree fairly well with values expected from the effect of pseudosynchronization (Hut 1981). For $e=0.125$, this rotation should be 1.09 times faster than the orbital rotation for $r \approx 0.29$ and $K_{1}+K_{2} \approx 420 \mathrm{~km} \mathrm{~s}^{-1}, v \sin i=133 \mathrm{~km} \mathrm{~s}^{-1}$.

As remarked in Sect. 4.3, the rate of apsidal advance derived from more recent, time-limited data only would imply a shorther apsidal period of 85.6 years - shorter than any earlier result. Notably, it is in better agreement with stellar models (Claret 2004). With the parameters from Table 10 and assuming that $k_{2}$ is identical for both components, $\log k_{2}$ can be calculated as -2.67 (the first solution gives -2.79 ). For a mass of $30 M_{\odot}$, a perfect agreement with the theoretical $\log k_{2}$ is obtained for a radius of $16.1 R_{\odot}$. However, due to the discrepancy between the evolutionary and observed masses, the published theoretical values of $k_{2}$ cannot reflect the real properties of the components properly.

These remaining inconsistencies demonstrate the need for more sophisticated evolutionary models of close binaries. It can only be hoped that future theoretical refinements and continuing observations might help to better understand this extraordinary and important O-type system.

Acknowledgements. Dr. H. Sana kindly put their individual RVs of V1007 Sco at our disposal. Our thanks are also due to Dr. B.D. Mason who informed us about unpublished speckle-interferometry of V1007 Sco, and to Dr. J. C. Mermilliod who clarified for us the problem of the correct HJDs for the CASLEO spectra. We gratefully acknowledge useful suggestions and a careful proofreading of the text by the referee, Dr. D. R. Gies. We greatly profitted from the use of the bibliographic services of CDS in Strasbourg and ADS of NASA. Research of P. Mayer, P. Harmanec and M. Wolf was supported from the research plan J13/98: 113200004 of Ministry of Education, Youth and Sports Investigation of the Earth and Universe. The research of the Czech authors was supported also from the grants GA ČR 205/03/0788, and GA ČR 205/06/0304 of the Czech Science Foundation and in final stages also from the Research Program MSM0021620860 Physical study of objects and processes in the solar system and in astrophysics of the Ministry of Education of the Czech Republic.
Table A.1. Parameters of HD 152218 and HD 152219. The rms errors of the last digits of individual elements are given in brackets.

\begin{tabular}{lcc}
\hline \hline Parameter & HD 152218 & HD 152219 \\
Spectral type & O9 V & O 9.5 IV \\
\hline$P_{\text {sider. }}$ day) & $5.60397(4)$ & $4.24028(3)$ \\
$T_{\text {peri. }}$ & $2453398.96(4)$ & $2453000.84(3)$ \\
$T_{\text {prim. min. }}$ & $2453398.87(1)$ & $2453000.23(1)$ \\
$e$ & $0.293(14)$ & $0.090(11)$ \\
$\omega($ deg $)$ & $102(3)$ & $150(2)$ \\
$\dot{\omega}\left(\mathrm{deg} . \mathrm{d}^{-1}\right)$ & $0.0051(5)$ & $0.0045(5)$ \\
$i(\mathrm{deg})$ & $68(1)$ & $72(3)$ \\
$K_{1}\left(\mathrm{~km} \mathrm{~s}^{-1}\right)$ & $156(5)$ & $109.3(5)$ \\
$K_{2}\left(\mathrm{~km} \mathrm{~s}^{-1}\right)$ & $219(10)$ & $284(4)$ \\
$\gamma\left(\mathrm{km} \mathrm{s}^{-1}\right)$ & $-26(2)$ & $-27(2)$ \\
$r_{1}$ & $0.21(\operatorname{assumed})$ & $0.37(2)$ \\
$r_{2}$ & $0.12($ assumed $)$ & $0.17(2)$ \\
$a \sin i\left(R_{\odot}\right)$ & $39.8(1.3)$ & $32.8(5)$ \\
$M_{1} \sin ^{3} i\left(M_{\odot}\right)$ & $15.7(9)$ & $19.1(6)$ \\
$M_{2} \sin ^{3} i\left(M_{\odot}\right)$ & $11.2(4)$ & $7.3(2)$ \\
$R_{1}\left(R_{\odot}\right)$ & $9.0(3)$ & $12.6(6)$ \\
$R_{2}\left(R_{\odot}\right)$ & $5.1(2)$ & $5.7(5)$ \\
$a\left(R_{\odot}\right)$ & $42.8(1.3)$ & $34.4(7)$ \\
$M_{1}\left(M_{\odot}\right)$ & $19.6(1.1)$ & $22.1(1.2)$ \\
$M_{2}\left(M_{\odot}\right)$ & $14.1(5)$ & $8.5(5)$ \\
\hline
\end{tabular}

\section{Appendix A: Ten eclipsing binaries in NGC 6231}

The cluster is known to contain a large number of spectroscopic binaries; García \& Mermilliod (2001) classified 16 as certain, and 14 as probable cases. Several others are listed by Stickland \& Lloyd (2001). There are also ten eclipsing binaries known in the cluster: V1034 Sco (CPD-41 ${ }^{\circ} 7742$ ) was studied by Sana et al. (2003), Bouzid \& Sterken (2004), and Sana et al. (2005); HD 152218, 152219, 326320, and CPD-41 ${ }^{\circ} 7746$ were discovered as eclipsing binaries by Otero \& Wils (2005a), Otero \& Wils (2005b), and Otero (2007) using ASAS-3 data (Pojmanski 2002); SBL 521 was discovered by Balona \& Engelbrecht (1985), SBL 334 by Balona \& Laney (1995) and SBL 314 and SBL 574 by Bouzid \& Sterken (2004). Periods of the last four objects are not yet known.

Recently, HD 152218 and 152219 were studied using the IUE spectra by Stickland et al. (1997) and by Stickland \& Lloyd (2001), respectively, and a comprehensive study of HD 152219 was published by Sana et al. (2006). Available RV measurements cover quite long time intervals in both cases: 53 years for HD 152218, and 36 years for HD 152219. It is, therefore, possible to derive the rate of apsidal advance for these systems. We used the ASAS-3 photometry from JD 2452455 to 2453600 (the older data are often unreliable for bright stars). Besides RVs noted by Sana et al. (2006) we included also RVs by Stickland \& Lloyd (2001) and Conti et al. (1977). Combined RV and light curve solutions were derived using the program FOTEL (Hadrava 1990, 2004a). For both systems, the radii from our solutions are rather uncertain. Since both binaries are well detached, there is no reason why the properties of their components should differ significantly from normal main-sequence stars. The uncertainty of both radii is larger in the case of HD 152218, so we assumed that the radii roughly agree with the values expected for stars of respective masses with an age of about $3 \times 10^{6}$ years. It is anticipated that the inclination obtained under such an assumption is fairly realistic. The solutions are listed in Table A.1.

There are no data for HD 326320 (B 0 V, period 1.317716) other than the ASAS-3 photometry. If the light curve is solved assuming $q=0.7$, then the inclination is $80^{\circ}$ and the relative 
radii of the components are 0.33 and 0.22 . Based on future observations hopefully more accurate results can be achieved for these eclipsing binaries. Due to their common cluster origin they will provide a unique opportunity to compare ages, masses and temperatures with evolutionary models.

\section{References}

Balona, L. A., \& Engelbrecht, C. A. 1985, MNRAS, 212, 889

Balona, L. A., \& Laney, C. D. 1995, MNRAS, 276, 627

Baume, G., Vázquez, R. A., \& Feinstein, A. 1999, A\&AS, 137, 233

Bouzid, M. Y., \& Sterken, C. 2004, in Spectroscopically and Spatially Resolving the Components of the Close Binary Stars, ed. R. W. Hilditch, H. Hensberge, \& K. Pavlovski, ASP Conf. Ser., 318, 288

Brownlee, R. R., \& Cox, A. N. 1953, ApJ, 118, 165

Claret, A. 2004, A\&A, 424, 919

Conti, P. S., Leep, E. M., \& Lorre, J. J. 1977, ApJ, 214, 759

Efron, B. 1979, Ann. Stat., 7, 1

Efron, B., \& Tibshirani, R. 1979, An Introduction to the Bootstrap (Chapman and Hall, London)

Feinstein, A., \& Ferrer, O. E. 1968, PASP, 80, 410

García, B., \& Mermilliod, J. C. 2001, A\&A, 368, 122

Hadrava, P. 1990, Contributions of the Astronomical Observatory Skalnate Pleso, 20, 23

Hadrava, P. 1995, A\&AS, 114, 393

Hadrava, P. 1997, A\&AS, 122, 581

Hadrava, P. 2004a, Publ. Astron. Inst. Acad. Sci. Czech Rep., 92, 1

Hadrava, P. 2004b, Publ. Astron. Inst. Acad. Sci. Czech Rep., 92, 15

Hadrava, P. 2005, Ap\&SS, 296, 239

Harmanec, P. 1998, A\&A, 335, 173

Harmanec, P., Horn, J., \& Juza, K. 1994, A\&AS, 104, 121

Hayford, P. 1932, Lick Observatory Bulletin, 16, 53

Heap, S. R., Lanz, T., \& Hubeny, I. 2006, ApJ, 638, 409

Hilditch, R. W. 2004, in Spectroscopically and Spatially Resolving the Components of the Close Binary Stars, ed. R. W. Hilditch, H. Hensberge, \& K. Pavlovski, ASP Conf. Ser., 318, 198

Hill, G., Crawford, D. L., \& Barnes, J. V. 1974, AJ, 79, 1271

Horn, J., Kubát, J., Harmanec, P., et al. 1996, A\&A, 309, 521
Howarth, I. D., Siebert, K. W., Hussain, G. A. J., \& Prinja, R. K. 1997, MNRAS, 284,265

Hut, P. 1981, A\&A, 99, 126

Lanz, T., \& Hubeny, I. 2003, ApJS, 146, 417

Levato, H., \& Morrell, N. 1983, Astrophys. Lett., 23, 183

Luna, H. G. 1988, A\&AS, 74, 427

Manfroid, J., Sterken, C., Cunow, B., et al. 1994, European Southern Observatory Scientific Report, 14, 1

Mason, B. D., Gies, D. R., Hartkopf, W. I., et al. 1998, AJ, 115, 821

Mayer, P., Lorenz, R., \& Drechsel, H. 1992, Informational Bulletin on Variable Stars, 3765,1

Mayer, P., Harmanec, P., Lorenz, R., et al. 2001, in The Influence of Binaries on Stellar Population Studies, ed. D. Vanbeveren, ASSL, 264, 567

Meynet, G., \& Maeder, A. 2000, A\&A, 361, 101

Otero, S. A. 2007, Open European Journal on Variable Stars, 72, 1

Otero, S. A., \& Wils, P. 2005a, Informational Bulletin on Variable Stars, 5630, 1 Otero, S. A., \& Wils, P. 2005b, Informational Bulletin on Variable Stars, 5644, 1 Penny, L. R., Gies, D. R., \& Bagnuolo, Jr., W. G. 1999, ApJ, 518, 450

Perryman, M. A. C., \& ESA 1997, The HIPPARCOS and TYCHO catalogues (The Hipparcos and Tycho catalogues. Astrometric and photometric star catalogues derived from the ESA Hipparcos Space Astrometry Mission (Noordwijk, Netherlands: ESA Publications Division), ESA SP Series, 1200 Pojmanski, G. 2002, Acta Astron., 52, 397

Prša, A., \& Zwitter, T. 2005, ApJ, 628, 426

Raboud, D., Cramer, N., \& Bernasconi, P. A. 1997, A\&A, 325, 167

Sana, H., Rauw, G., \& Gosset, E. 2001, A\&A, 370, 121

Sana, H., Hensberge, H., Rauw, G., \& Gosset, E. 2003, A\&A, 405, 1063

Sana, H., Antokhina, E., Royer, P., et al. 2005, A\&A, 441, 213

Sana, H., Gosset, E., \& Rauw, G. 2006, MNRAS, 371, 67

Škoda, P. 1996, in Astronomical Data Analysis Software and Systems V, ed. G. H. Jacoby, \& J. Barnes, ASP Conf. Ser., 101, 187

Stickland, D. J., \& Lloyd, C. 2001, The Observatory, 121, 1

Stickland, D. J., Lloyd, C., \& Penny, L. R. 1997, The Observatory, 117, 213

Stickland, D. J., Lloyd, C., Penny, L. R., Gies, D. R., \& Bagnuolo, Jr., W. G. 1996, The Observatory, 116, 226

Struve, O. 1944, ApJ, 100, 189

Sung, H., Bessell, M. S., \& Lee, S.-W. 1998, AJ, 115, 734

van Hamme, W. 1993, AJ, 106, 2096

Walborn, N. R. 1972, AJ, 77, 312

Wilson, R. E., \& Devinney, E. J. 1971, ApJ, 166, 605 